\title{
Effect of radiotherapy on the treatment of patients with extensive stage small cell lung cancer
}

\author{
C. Wu, T. Wang, J. Wang, B. Qu, H. Wang and Y. Hu \\ Department of Oncology, \\ The General Hospital of the People's Liberation Army, Beijing, China \\ Corresponding author: Y. Hu \\ E-mail:wcwhcn@163.com
}

Genet. Mol. Res. 13 (4): 8577-8585 (2014)

Received May 10, 2013

Accepted October 25, 2013

Published January 24, 2014

DOI http://dx.doi.org/10.4238/2014.January.24.7

\begin{abstract}
Small cell lung cancer (SCLC) is an aggressive disease, in which more than $80 \%$ of patients present distant metastases at the time of first diagnosis. Chemotherapy is considered as the main treatment of extensive disease of SCLC (ED-SCLC), while the role of radiotherapy in the treatment of ED-SCLC is controversial. A case-control study was undertaken of patients diagnosed as ED-SCLC between 2004 and 2010. Fifty-eight patients with overall survival (OS) over 1 year were chosen, and another 58 patients with OS less than 1 year were selected as the control group, with the age, gender, metastasis or no metastasis of the liver, and the response after the first line of chemotherapy matched. The 1-year, 2-year, 3-year, and median OS of the 75 ED-SCLC patients who received radiation were $81.47,29.89,22.77 \%$, and 17 months, respectively, and were $61.18,23.53,0 \%$, and 16 months, respectively, for patients who did not receive radiation. The $\chi^{2}$ test and odds ratio (OR) estimate demonstrated that these differences were statistically significant $\left(\chi^{2}=6.38, \mathrm{P}=0.0116 ; \mathrm{OR}=2.74,95 \%\right.$ confidence interval $\left.=1.24-6.05\right)$. These results show that radiotherapy also plays a role in responding patients with extensive stage of SCLC.
\end{abstract}

Key words: Small cell lung cancer; Extensive stage; Radiotherapy 


\section{INTRODUCTION}

The incidence of lung cancer has been increasing in recent years. Small cell lung cancer (SCLC), which is expected to account for approximately $15-20 \%$ of all new cases of lung cancers, is characterized by an aggressive clinical course and shows high sensitivity to both chemotherapy and radiotherapy. SCLC is clinically categorized at two stages: limited disease (LD) and extensive disease (ED). At the time of first diagnosis, only $40 \%$ of patients present LD-SCLC (Govindan et al., 2006). Despite the high response rate to initial chemotherapy and radiotherapy, most patients subsequently experience a relapse of the primary tumor or distant metastasis. The overall survival rate is low. The standard therapy for LD-SCLC is concurrent chemoradiotherapy, and the 5 -year survival rate observed in clinical trials is approximately $25 \%$. The treatment for ED-SCLC is mainly chemotherapy. Therefore, the role of radiotherapy in the treatment for patients with extensive stage SCLC is currently disputed. Our case-control study evaluated the role of radiotherapy in the treatment of ED-SCLC through an analysis of 116 ED-SCLC patients in order to provide data for evidenced-based medicine for clinical research.

\section{MATERIAL AND METHODS}

A review was undertaken of patients who were diagnosed with SCLC between January 2004 and January 2010 at the General Hospital of the People's Liberation Army (PLAGH). Informed consent was obtained from all patients of our group, and our case-control study was conducted with the approval of the Ethics Committee and our hospital. Fifty-eight EDSCLC patients with overall survival (OS) over 1 year were chosen, and an equal number of ED-SCLC patients with OS less than 1 year were selected as the control group, with the age, gender, metastasis or no metastasis of the liver, and the response after the first-line chemotherapy matched. A uniform set of pretreatment-staging procedures was carried out routinely, including brain magnetic resonance imaging and chest computed tomography (CT) scans, abdominal ultrasound or CT scan, bone scan, and ultrasonographic diagnosis of the cervical lymph nodes. Based on the above results of staging, patients were classified into two stages: LD- and ED-SCLC. LD was defined as disease confined to the thorax, including the bilateral mediastinal and supraclavicular nodes. Involvement beyond these confines was defined as ED. Patients with ipsilateral pleural effusion and patients with bilateral mediastinal and supraclavicular lymph node metastasis were classified as ED, whereas those with ipsilateral mediastinal and supraclavicular lymph node metastasis were classified as LD. All patients in our group were confirmed to be in the extensive stage.

These patients received a platinum-based chemotherapy regimen, usually containing etoposide and cisplatin (a platinum agent) (EP), etoposide and carboplatin (EC), and irinotecan and etoposide (IP), and some patients were given epirubicin, cisplatin, and etoposide (AEP). The firstline chemotherapy was usually EP, EC, or IP; only a few of the patients received the AEP regimen. Some patients received topotecan instead of irinotecan, teniposide instead of etoposide, or nedaplatin instead of cisplatin. The patients in our study were given an overall checkup every two cycles of chemotherapy. The following therapeutic evaluations were recorded: complete response (CR), partial response (PR), stable disease, or progression of disease, according to the Response Evaluation Criteria in Solid Tumors. Some of the patients received palliative thoracic radiotherapy (TRT) and some received radiotherapy to the metastatic mediastinal and supraclavicular lymph nodes or 
bone. Prophylactic cranial irradiation (PCI) was limited to only a few patients. Telephone followup was carried out for the survival period from the first day of confirmation of diagnosis until December 2010. All statistical analyses were conducted using the SAS 9.2 statistical software (SAS Institute, Inc., Cary, NC, USA) using the Pearson $\chi^{2}$ test or the Fisher exact test for nominal variables and the Student $t$-test for continuous variables. Statistical significance was assumed for a two-tailed $\mathrm{P}$ value less than 0.05 . Baseline patient characteristics are shown in Table 1.

Table 1. Baseline patient characteristics in the two groups.

\begin{tabular}{|c|c|c|c|c|}
\hline & \multicolumn{2}{|c|}{ Overall survival $<1$ year } & \multicolumn{2}{|c|}{ Overall survival $>1$ year } \\
\hline & Number & Percent (\%) & Number & Percent (\%) \\
\hline \multicolumn{5}{|l|}{ Age of diagnosis } \\
\hline$\geq 60$ years & 26 & 44.83 & 26 & 44.83 \\
\hline$<60$ years & 32 & 55.17 & 32 & 55.17 \\
\hline \multicolumn{5}{|l|}{ Gender } \\
\hline Male & 54 & 93.10 & 54 & 93.10 \\
\hline Female & 4 & 6.90 & 4 & 6.90 \\
\hline \multicolumn{5}{|l|}{ Smoking status } \\
\hline Yes & 47 & 81.03 & 46 & 79.31 \\
\hline No & 11 & 18.97 & 12 & 20.69 \\
\hline \multicolumn{5}{|c|}{ Karnofsky performance status } \\
\hline$>80$ & 56 & 98.25 & 56 & 96.55 \\
\hline$<80$ & 1 & 1.75 & 2 & 3.45 \\
\hline \multicolumn{5}{|l|}{ Type of pathology } \\
\hline Pure & 58 & 100.00 & 57 & 98.28 \\
\hline Mixed & 0 & 0.00 & 1 & 1.72 \\
\hline \multicolumn{5}{|l|}{ Liver metastasis or not } \\
\hline Yes & 12 & 20.69 & 12 & 20.69 \\
\hline No & 46 & 79.31 & 46 & 79.31 \\
\hline \multicolumn{5}{|c|}{ Response of first-line hemotherapy } \\
\hline Complete response & 1 & 1.72 & 1 & 1.72 \\
\hline Partial response & 44 & 75.86 & 44 & 75.86 \\
\hline Stable disease & 10 & 17.24 & 10 & 17.24 \\
\hline Progression of disease & 3 & 5.17 & 3 & 5.17 \\
\hline \multicolumn{5}{|l|}{ Hemoglobin } \\
\hline Normal & 48 & 88.89 & 49 & 89.09 \\
\hline Anemia & 6 & 11.11 & 6 & 10.91 \\
\hline \multicolumn{5}{|l|}{ Albumin } \\
\hline Normal & 49 & 89.09 & 51 & 91.07 \\
\hline Hypoproteinemia & 6 & 10.91 & 5 & 8.93 \\
\hline \multicolumn{5}{|l|}{ Lactate dehydrogenase } \\
\hline Normal & 38 & 70.37 & 43 & 76.79 \\
\hline Elevated & 16 & 29.63 & 13 & 23.21 \\
\hline \multicolumn{5}{|l|}{ Neuron-specific enolase } \\
\hline Normal & 11 & 21.15 & 15 & 27.27 \\
\hline Elevated & 41 & 78.85 & 40 & 72.73 \\
\hline \multicolumn{5}{|l|}{ Carcinoembryonic antigen } \\
\hline Normal & 25 & 48.08 & 34 & 61.82 \\
\hline Elevated & 27 & 51.92 & 21 & 38.18 \\
\hline \multicolumn{5}{|c|}{ CYFRA21-1 (cytokeratin 19 fragment) } \\
\hline Normal & 34 & 65.38 & 40 & 75.47 \\
\hline Elevated & 18 & 34.62 & 13 & 24.53 \\
\hline \multicolumn{5}{|l|}{ Platelet } \\
\hline Normal & 43 & 79.63 & 41 & 77.36 \\
\hline Elevated & 11 & 20.37 & 12 & 22.64 \\
\hline \multicolumn{5}{|l|}{ Leucopenia } \\
\hline Grade $1-2$ & 41 & 74.55 & 45 & 80.36 \\
\hline Grade 3-4 & 14 & 25.45 & 11 & 19.64 \\
\hline
\end{tabular}

The above baseline patient characteristics in the two groups were not statistically different $(\mathrm{P}>0.05)$. 


\section{RESULTS}

The 1-year, 2-year, 3-year, and median OS of the 75 ED-SCLC patients who received radiation were $81.47,29.89,22.77 \%$, and 17 months, respectively, and were $61.18,23.53$, $0 \%$, and 16 months, respectively, for patients who did not. In the radiation therapy group, the survival time was shorter than 1 year for 31 patients $(41.33 \%)$, but was longer than 1 year for 44 patients $(58.67 \%)$. In the no radiation therapy group, the survival time was shorter than 1 year for 27 patients $(65.85 \%)$, but was longer than 1 year for 14 patients $(34.15 \%)$, as shown in Figure 1. The differences in survival were significant between the two groups $\left(\chi^{2}=6.38, \mathrm{P}\right.$ $=0.0116$; odds ratio $(\mathrm{OR})=2.74,95 \%$ confidence interval $=1.24-6.05)$.

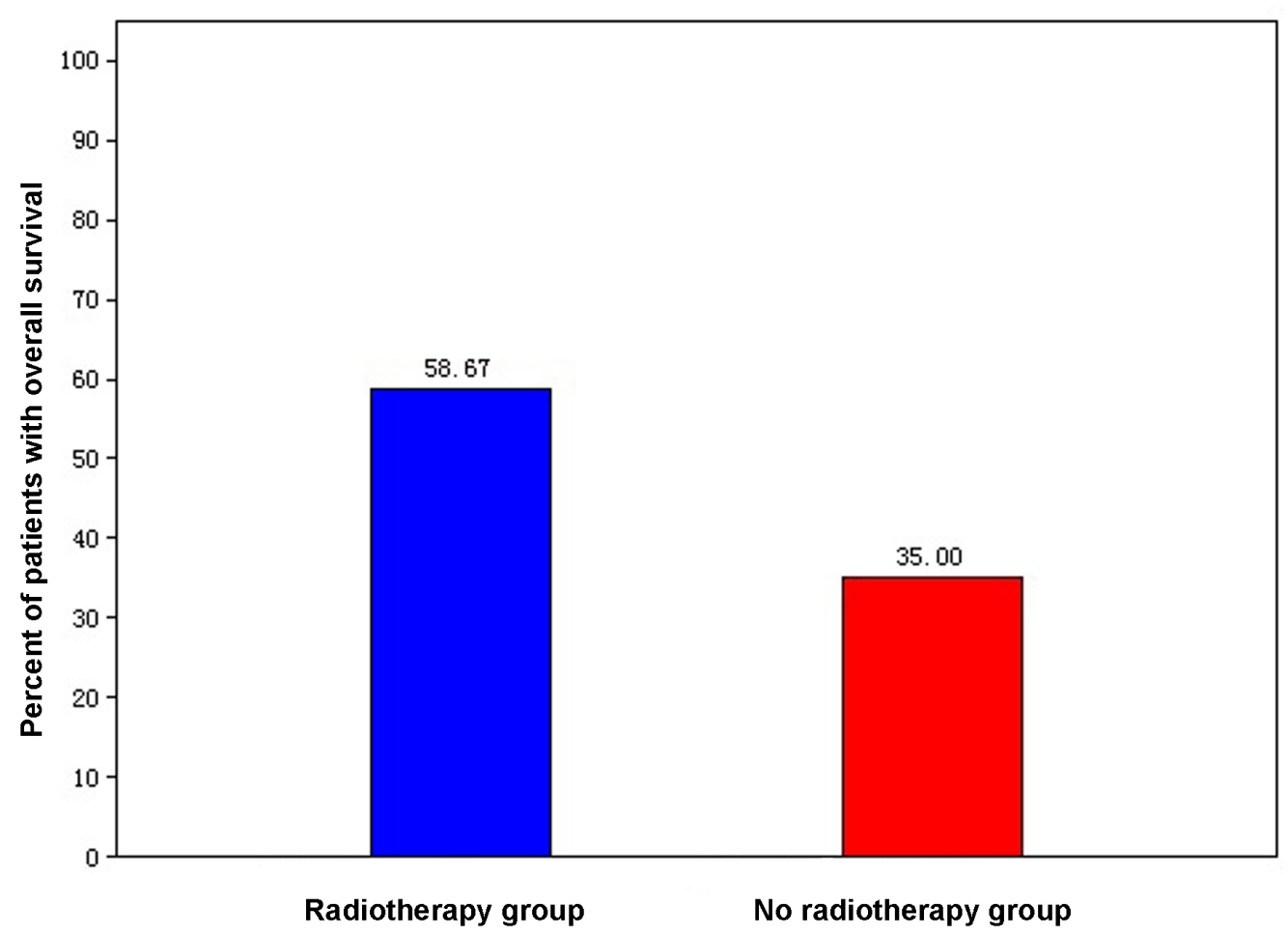

Figure 1. Role of radiotherapy in the treatment of ED-SCLC.

\section{DISCUSSION}

\section{Role of radiotherapy in the treatment of ED-SCLC}

SCLC is divided into the LD and the ED according to the Veterans Administration Lung Cancer Study Group Classification. LD-SCLC is defined as a disease that can be encompassed within a reasonable radiation field, and ED-SCLC is defined as a disease that is greater than a reasonable radiation field. The major treatment for ED-SCLC is chemotherapy. Despite 
the high response rate to chemotherapy with $>50 \%$ confirmed objective responses, the majority of patients relapse within several months after first-line chemotherapy, with the usual form of thoracic relapse and cranial metastasis. Worse still, further treatment or medicine is to limited effect, and the response rate to the second-line chemotherapy is very low, so that patients usually die of uncontrolled tumor growth. The data from the analysis of surveillance, epidemiological, and end results (SEER) showed that in 2000, the 2-year survival rate for patients with ED-SCLC was only 4.6\%, which increased from a rate of $1.5 \%$ in 1973 (Govindan et al., 2006).

Patterns of failure in patients with ED-SCLC treated with chemotherapy alone show that besides distant progression, local progression remains a very frequent event. Therefore, TRT and/or PCI could be of benefit for some patients. Patients most likely to experience some form of response to chemotherapy, achieving CR or PR in the first-line treatment, which forms the basis for overall control of disease progression, have reasonable chances of prolonged periods of survival.

In addition to alleviating the symptoms of cranial metastasis or ostalgia, TRT can be used as consolation, yielding a higher probability of local control with a lower incidence of recurrence. Approximately one-fifth of patients show evidence of cranial metastasis at diagnosis. Patients are at high risk of cranial metastasis during the treatment process, and about one half of the limited stage patients will develop de novo cranial metastasis after complete remission of the original foci (Arriagada et al., 1995). Patients with advanced disease have a higher incidence of cranial involvements. However, most of the chemotherapeutic agents cannot penetrate the blood-brain barrier. Recent reports have demonstrated that PCI can further improve survival. The randomized phase III trial performed by the EORTC (08993-22993) compared PCI to observations in patients with stable disease of response to first-line chemotherapy. PCI was associated with a considerably reduced risk of developing symptomatic brain metastases and with improved survival; the 1-year survival after randomization was 27.1 vs $13.3 \%(\mathrm{P}=$ 0.003) (Slotman et al., 2007). Meta-analyses have confirmed this result and showed that PCI resulted in improved survival (Meert et al., 2001). Therefore, PCI is proposed for patients with disease stabilization or remission after first-line chemotherapy, and it is expected to improve survival with the use of thoracic irradiation by reducing the rate of local relapses in chemotherapy responders.

Despite the compelling data that PCI reduces the incidence of brain metastasis and improves overall survival in SCLC, its indications should also be considered in light of its potential neurotoxicity. Several studies have reported neurological and intellectual impairment or abnormalities on brain CT scan that are potentially related to PCI, which can be a concern to clinicians. Acute toxicity is generally manageable and consists mostly of alopecia, headaches, fatigue, nausea, and vomiting. Long-term toxicity is worthy of attention, since sequelae, such as severe memory loss, intellectual impairment, or even dementia, ataxia, or seizures, have been reported in retrospective studies and have been attributed to PCI, which was carried out in a few patients of our study.

The question of whether or not TRT also plays a role in responding patients with EDSCLC is not yet resolved. There is only one study that systematically addressed the role of TRT in patients with ED-SCLC. Jeremic et al. (1999) treated 206 patients with 3 cycles of chemotherapy consisting of cisplatin and etoposide. Patients $(\mathrm{N}=109)$ with a complete response at distant sites and a complete or partial response in the thorax were randomized to either the chemotherapy alone group or the accelerated hyper-fractionated radiotherapy group. Patients 
received radiotherapy of $54 \mathrm{~Gy}$ in 36 fractions over 18 days along with carboplatin and topside (Jeremic et al., 1999). TRT was also given to all patients who had PR at distant sites, without randomization. The use of TRT in patients with a CR at distant sites gave rise to significant improvement of survival. Median survival was 17 months for patients who received TRT, compared to only 11 months for those who received chemotherapy alone (Jeremic et al., 1999). The 5-year survival rate was $9.1 \%$ for the TRT group and was $3.7 \%$ for the chemotherapy-only group $(\mathrm{P}=0.041)$ (Jeremic et al., 1999). However, this one small-, single-center randomized study has not led to the routine use of TRT in ED-SCLC.

Most of the patients in our study received palliative TRT, with once daily escalated thoracic radiation of $66 \mathrm{~Gy}-50 \mathrm{~Gy} / 33-25$ fractions. A few patients received palliative radiotherapy to alleviate the symptoms of cranial metastasis and ostalgia, whereas PCI was scarcely used with 20-30 Gy in 10 fractions.

This case-control study showed that in the radiation therapy group, the percentage of patients with survival times longer than 1 year was $58.67 \%$, while in the other group, it was only $34.15 \%$, which was significantly lower than the former $(\mathrm{P}=0.0116)$. The OR value suggested that the group receiving radiation therapy was 2.74 times more likely to survive one year compared to the other group.

\section{Chemotherapy regimen in combination with radiation therapy}

Because of the high propensity of SCLC to metastasize, chemotherapy is the pivotal treatment. Response rates are high, with $50-90 \%$ of patients showing confirmed partial or complete responses. ED-SCLC patients should be treated with cisplatin or carboplatin in combination with etoposide for four to six cycles, which is recommended in the National Comprehensive Cancer Network (NCCN, 2011) guidelines for category IIA.

In the 1970s, the commonly used chemotherapy regimen combined cyclophosphamide, anthracycline, and vincristine (CAV). However, cyclophosphamide and anthracycline could rarely be administered concurrently with radiotherapy. Since the early 1980s, a very popular regimen in SCLC has combined EP, or EC if patients cannot be given cisplatin (Simon and Turrisi, 2007; Olszewski et al., 2010). A phase III study confirmed that chemotherapy with EP was better than CAV (Sundstrom et al., 2002). For patients with limited disease, the 2- and 5-year survival rates reached 25 and $10 \%$, respectively, in the EP group, which compared favorably with the rates of 8 and 3\%, respectively, in the cyclophosphamide, epirubicin and vincristine group. The meta-analysis of Pujol et al. (2000), which was derived from approximately 4000 patients enrolled in 19 published randomized trials, revealed a higher OS rate with a cisplatincontaining regimen, and demonstrated the general effectiveness of cisplatin in SCLC.

In recent years, modern topoisomerase-I inhibitors, such as topotecan or irinotecan, have provided new hope for the treatment of SCLC. One of the most controversial questions in the treatment of ED-SCLC is whether topotecan or irinotecan is superior to etoposide. Two prospectively randomized phase III trials compared topotecan + cisplatin to etoposide + cisplatin treatments. The North American trial randomized 784 patients to the oral topotecan + cisplatin group and the intravenous etoposide + cisplatin group. This trial showed the non-inferiority of the topotecan/cisplatin combination, and a slightly but statistically longer progression-free survival with etoposide/cisplatin (Eckardt et al., 2006; Lara Jr. et al., 2009). The second study demonstrated significant improvement of the response rate and progression- 
free survival in support of intravenous topotecan/cisplatin. However, the primary end point of the study was OS, which showed no difference between the groups. Hematological toxicity was higher in the topotecan group (Heigener et al., 2008). Since no overall survival advantage could be revealed, topotecan has not become a standard first-line treatment.

In the 116 patients included in this study, the first-line chemotherapy was mainly EC or EP. A few patients were given the IP regimen as the first-line chemotherapy, although this was mostly used as the second-line chemotherapy. Fifty-four patients changed the regimen into second-line chemotherapy because of the failure of the first-line chemotherapy, whereas the other 62 patients gave up further chemotherapy.

\section{Modalities and timing of radiotherapy}

There are currently different ways of combining treatment modalities: whether to administer TRT and chemotherapy concurrently or sequentially, and whether radiation should be administered early or late in the overall course of treatment continue to be subjects of debate. Some studies have shown that better results have been obtained with a radiotherapy concomitant with chemotherapy regimen in terms of the survival rate, which has become the most popular regimen (Turrisi III et al., 1999; Takada et al., 2002). Concomitant approaches have the advantage of shortening the whole duration of treatment time, but at an increased risk of acute toxicities, especially esophageal. Clinicians are now expected to manage these toxicities. However, some studies have not found any difference between the two modalities with respect to the OS.

Although the timing of radiation therapy continues to be debated, the 2011 NCCN guidelines in SCLC state that there is category 1 evidence to support concurrent chemoradiotherapy over sequential therapy for fit patients (Stinchcombe and Gore, 2010; Komatsu et al., 2011; NCCN, 2011).

Moreover, several trials compared combined modality treatments in which radiotherapy was administered either early or late. The results of a meta-analysis (De Ruysscher et al., 2006) showed that the interval between the "start of any treatment and the end of radiotherapy" (SER) should be as short as possible. Patients with SE $<30$ days had a predicted better 5 -year survival rate that was greater than $20 \%$. The effect of sequential TRT could be reduced by prolonged chemotherapy, which might result in the regeneration of drug-resistant tumor cells (Brade and Tannock, 2006; Combs et al., 2012).

No clinical studies are currently available with respect to the effect of "combining treatment modalities in ED-SCLC" and its initiating time. Early intervention by radiotherapy may interfere with the evaluation of chemotherapy and impair tolerance. However, late intervention will reduce the chance of local control and lead to resistance of chemotherapy. In clinical practice, the choice of the model and initiating time are determined for each individual. For example, some patients with immense thoracic mass tumors that are beyond the range of radiotherapy will receive 2 to 4 cycles of chemotherapy before regional radiotherapy. For patients with poor performance or severe hematological toxicities, "the concurrent chemoradiotherapy" regimen is generally avoided. In the present cohort, radiotherapy was initiated after 3 to 5 cycles of chemotherapy in most patients. In some other patients, radiotherapy was given at a later stage as palliative therapy to control the symptoms of cranial metastasis or ostalgia. Overall, in advanced diseases, the time and model of radiotherapy cannot be predetermined, and should be determined individually. 


\section{Treatment-related toxicity}

In our study, esophagitis occurred in few patients who received radiotherapy, a few patients developed pneumonia, but the incidence rate was very low, and no patients died of radiotherapy-related toxicity. The III-IV grade hematological toxicity, such as leucopenia, showed no significant difference, indicating that in our group, radiation therapy did not obviously increase hematological toxicity. In conclusion, radiotherapy has a relatively good tolerance and can be manageable.

\section{CONCLUSIONS}

For the ED-SCLC patients, the aim of therapy is to improve the quality of life and to prolong the survival duration. The major treatment is chemotherapy, but patterns of failure in patients with ED-SCLC treated with chemotherapy alone have shown that besides distant progression, local progression remains a very frequent event. Therefore, we believe that the effect of radiotherapy should not be ignored. On the one hand, there is enough evidence demonstrating that PCI may result in improved survival; however, the role of TRT remains uncertain. We believe that TRT could be of benefit for suitable patients. The results of our case-control study further confirmed the role of radiation therapy in prolonging survival in ED-SCLC after sufficient chemotherapy. Our present study is only retrospective, and no similar perspective study is currently available. It is expected that the results of larger trials confirming this hypothesis will be obtained in forthcoming years.

\section{REFERENCES}

Arriagada R, Le Chevalier T, Borie F, Riviere A, et al. (1995). Prophylactic cranial irradiation for patients with small-cell lung cancer in complete remission. J. Natl. Cancer Inst. 87: 183-190.

Brade AM and Tannock IF (2006). Scheduling of radiation and chemotherapy for limited-stage small-cell lung cancer: repopulation as a cause of treatment failure? J. Clin. Oncol. 24: 1020-1022.

Combs SE, Zipp L, Rieken S, Habermehl D, et al. (2012). In vitro evaluation of photon and carbon ion radiotherapy in combination with chemotherapy in glioblastoma cells. Radiat. Oncol. 7: 9.

De Ruysscher D, Pijls-Johannesma M, Bentzen SM, Minken A, et al. (2006). Time between the first day of chemotherapy and the last day of chest radiation is the most important predictor of survival in limited-disease small-cell lung cancer. J. Clin. Oncol. 24: 1057-1063.

Eckardt JR, von Pawel J, Papai Z, Tomova A, et al. (2006). Open-label, multicenter, randomized, phase III study comparing oral topotecan/cisplatin versus etoposide/cisplatin as treatment for chemotherapy-naive patients with extensive-disease small-cell lung cancer. J. Clin. Oncol. 24: 2044-2051.

Govindan R, Page N, Morgensztern D, Read W, et al. (2006). Changing epidemiology of small-cell lung cancer in the United States over the last 30 years: analysis of the surveillance, epidemiologic, and end results database. J. Clin. Oncol. 24: 4539-4544.

Heigener DF, Freitag L, Eschbach C and Huber RM (2008). Topotecan/cisplatin (TP) compared to cisplatin/etoposide (PE) for patients with extensive disease-small cell lung cancer (ED- SCLC): final results of a randomized phase III trial. J. Clin. Oncol. 26: 7513.

Jeremic B, Shibamoto Y, Nikolic N, Milicic B, et al. (1999). Role of radiation therapy in the combined-modality treatment of patients with extensive disease small-cell lung cancer: A randomized study. J. Clin. Oncol. 17: 2092-2099.

Komatsu T, Oizumi Y, Kunieda E, Tamai Y, et al. (2011). Definitive chemoradiotherapy of limited-disease small cell lung cancer: Retrospective analysis of new predictive factors affecting treatment results. Oncol. Lett. 2: 855-860.

Lara PN Jr, Natale R, Crowley J, Lenz HJ, et al. (2009). Phase III trial of irinotecan/cisplatin compared with etoposide/ cisplatin in extensive-stage small-cell lung cancer: clinical and pharmacogenomic results from SWOG S0124. $J$. Clin. Oncol. 27: 2530-2535. 
Meert AP, Paesmans M, Berghmans T, Martin B, et al. (2001). Prophylactic cranial irradiation in small cell lung cancer: a systematic review of the literature with meta-analysis. BMC Cancer 1: 5.

National Comprehensive Cancer Network (2011). NCCN Clinical Practice Guidelines in Oncology: Small Cell Lung Cancer. Vol. 2.

Olszewski U, Poulsen TT, Ulsperger E, Poulsen HS, et al. (2010). In vitro cytotoxicity of combinations of dichloroacetate with anticancer platinum compounds. Clin. Pharmacol. 2: 177-183.

Pujol JL, Carestia L and Daures JP (2000). Is there a case for cisplatin in the treatment of small-cell lung cancer? A metaanalysis of randomized trials of a cisplatin-containing regimen versus a regimen without this alkylating agent. $B r$. $J$. Cancer 83: 8-15.

Simon GR and Turrisi A (2007). Management of small cell lung cancer: ACCP evidence-based clinical practice guidelines (2nd edn.). Chest 132: 324S-339S.

Slotman B, Faivre-Finn C, Kramer G, Rankin E, et al. (2007). Prophylactic cranial irradiation in extensive small-cell lung cancer. N. Engl. J. Med. 357: 664-672.

Stinchcombe TE and Gore EM (2010). Limited-stage small cell lung cancer: current chemoradiotherapy treatment paradigms. Oncologist 15: 187-195.

Sundstrom S, Bremnes RM, Kaasa S, Aasebo U, et al. (2002). Cisplatin and etoposide regimen is superior to cyclophosphamide, epirubicin, and vincristine regimen in small-cell lung cancer: results from a randomized phase III trial with 5 years' follow-up. J. Clin. Oncol. 20: 4665-4672.

Takada M, Fukuoka M, Kawahara M, Sugiura T, et al. (2002). Phase III study of concurrent versus sequential thoracic radiotherapy in combination with cisplatin and etoposide for limited-stage small-cell lung cancer: results of the Japan Clinical Oncology Group Study 9104. J. Clin. Oncol. 20: 3054-3060.

Turrisi AT III, Kim K, Blum R, Sause WT, et al. (1999). Twice-daily compared with once-daily thoracic radiotherapy in limited small-cell lung cancer treated concurrently with cisplatin and etoposide. N. Engl. J. Med. 340: 265-271. 